\title{
24 Umgang mit Fehlern
}

\author{
Thomas Fleischmann
}

It's human to err, to forgive is divine. Neither is Marine policy.

Motto des United States Marine Corps

\subsection{Einleitung}

Was hat dieser etwas rüde Satz mit dem Umgang mit Fehlern in der Medizin, speziell der Notfallmedizin, zu tun? Vielleicht etwas mehr, als man gern hätte, denn der Umgang mit Fehlern in der Medizin unterscheidet sich bei nüchterner Betrachtung manchmal gar nicht so sehr von der Einstellung des United States Marine Corps. In beiden Bereichen nimmt man für sich in Anspruch, ziemlich fehlerfrei vorzugehen, und in beiden Bereichen werden Fehler eher nicht verziehen, auch wenn dies ethisch noch so hochstehend wäre.

Auch wenn in den letzten Jahren im Gesundheitswesen ein neues Bewusstsein im Umgang mit Fehlern wächst, so findet sich immer noch die Personalisierung von Fehlern als eine nicht selten ergriffene Reaktionsweise, auch wenn dies ethisch und sachlich oft falsch ist. Ein vermeintlich Schuldiger wird dann manchmal schnell gefunden oder bestimmt. Jenem Menschen wird die Schuld am Geschehen mit all seinen Folgen aufgeladen, was gleichzeitig zur Entlastung aller anderen Beteiligten dient, die es immer auch gibt (Reason 200o). So vorzugehen ähnelt sehr dem bereits im Buch Moses beschriebenen Sündenbock-Prinzip.

Das Personalisieren von Schuld ist eine offenbar tief im menschlichen Wesen wurzelnde Reaktion auf Fehler. Diese Denkweise mag psychologisch verständlich sein, sie ist aber nicht nur unethisch, sondern oft auch sachlich falsch, denn in der Mehr- 
zahl der Fälle steht hinter der vermeintlich persönlichen Schuld eines Einzelnen das Versagen eines Systems (Reason 2000).

\section{Es ist sehr viel einfacher und offenbar auch befriedigender, einem Einzelnen die Schuld zu geben als einem System (Reason 2000). Es ist aber sachlich meis- tens falsch, und es ist unethisch.}

Es gibt vor allem zwei bedenkliche Folgen der Personalisierung von Schuld: die Traumatisierung vermeintlich Schuldiger und das Unterlassen der Maßnahmen, die die Wiederholung des Fehlers vermeiden können. Im ersteren Fall geschieht dem vermeintlich Schuldigen Unrecht, im zweiten können künftige Patienten zu Schaden kommen. Beides ist ethisch nicht vertretbar.

Helfer, oft Ärzte, denen die Schuld an einem Fehler zugeschoben wird, leiden oft sehr (Wulf 2013). Gerade bei verantwortungsbewussten und hilfsbereiten Menschen mit hoher Motivation zum Helfen besteht nach einem Fehler oft eine erhebliche Bereitschaft, die Ursache tatsächlich bei sich zu sehen. Dies lässt sich manchmal selbst dann beobachten, wenn dies sachlich gar nicht richtig oder zumindest nicht angemessen ist. In diesen Fällen leidet dann unter Umständen nicht die Person mit dem höchsten Anteil am Geschehen, sondern die mit dem höchsten Verantwortungsgefühl. Die menschlich berührende, bei sachlicher Betrachtung oft aber zu relativierende Bereitschaft mancher Helfer, die Schuld bei sich zu suchen, kann dann unter ungünstigen Umständen auf eine nur zu gern schuldzuweisende Umgebung treffen. Manchmal bleibt die Wahrheit dann auf der Strecke und die Chance, aus einem Fehler zu lernen, wird vertan.

Die zweite ethisch und sachlich bedenkliche Folge der Personalisierung von Fehlern besteht darin, dass durch die Verschiebung auf eine Person der Blick davon abgewendet wird, dass hinter dem fehlerhaften Handeln eines Menschen oft in Wirklichkeit das Versagen eines Systems steht (Reason 200o). Wird dies nicht erkannt und demzufolge nicht auf der Systemebene gehandelt, dann ist die Gefahr groß, dass sich der Fehler wiederholt. Umgekehrt kann die Wahrscheinlichkeit des Wiederauftretens eines Fehlers meist eher dadurch verringert werden, dass auf der Systemebene Änderungen vollzogen werden als dass Personen ausgetauscht werden (Reason 200o). Somit gebieten nicht nur der ethische, sondern auch der sachliche und der präventive Umgang mit einem Fehler in der Medizin eine Abwendung von der PersonenEbene und eine Hinwendung zur System-Ebene (Rall et al. 2001).

Auch wenn beim Umgang mit Fehlern in der Medizin bisher vom Verhalten der Helfer die Rede war, so darf nie aus dem Licht der Aufmerksamkeit geraten, dass immer das Leid der Patienten im Mittelpunkt stehen muss. Sie sind es, an denen sich der Fehler verwirklicht, und sie müssen mit den Folgen eines Fehlers körperlich und seelisch leben. Es scheint in der conditio humana zu liegen, dass Schäden, die durch einen anderen Menschen zugefügt werden, wenn auch nicht willentlich, als viel schwerer empfunden werden, als wenn sie als schicksalhaft, als von außen kommend und unvermeidbar angesehen werden. Besonders schwer ist das Ertragen von Schäden durch Fehler, wenn sie von Menschen begangen werden, denen man nur zu gern vertraut oder vertrauen möchte, zum Beispiel Ärzten, Pflegekräften oder Mitarbeitern des Rettungsdienstes. 
Beim Umgang mit Fehlern in der Medizin zeigen sich vor allem folgende ethische Aspekte:

- Der Patient und seine Angehörigen haben ein ethisches (und juristisches) Recht, über das Geschehen angemessen und empathisch aufgeklärt zu werden.

- Die betroffenen Helfer haben das ethische Recht, angemessen und empathisch behandelt zu werden, auch von ihren Mitarbeitern und Vorgesetzten.

- Künftige Patienten haben ein ethisches Recht, dass Fehler sachlich analysiert und Maßnahmen ergriffen werden, die ihre Wiederholung vermeiden.

Diese ethischen Ansprüche entsprechen den Werten:

- Ehrlichkeit für betroffene Patienten

- Gerechtigkeit für betroffene Helfer

- Schutz für künftige Patienten

\section{Fallbeispiel 1: Gescheiterte Intubation und die Folgen}

Dr. S., ein junger, engagierter und hilfsbereiter Arzt im ersten Jahr der Weiterbildung zum Internisten, übernimmt für einen Kollegen auf dessen Bitte hin einen Nachtdienst in der Notaufnahme. Es handelt sich um einen seiner ersten Nachtdienste dort. Gegen 2 Uhr wird Frau H. eingeliefert, 36 lahre alt und alleinerziehende Mutter von zwei Kindern, da sie an einem schweren Asthma-Anfall leidet. Die von Dr. S. korrekt durchgeführte medikamentöse Therapie schlägt nicht an. Schließlich dekompensiert die Patienten rasch, wird zyanotisch und ist nicht mehr ansprechbar. Dr. S. versucht die nun hypoxische und bewusstlose Frau zu intubieren, was ihm nicht gelingt. Eine Beatmung mit Beutel und Maske scheitert am hohen Beatmungsdruck. Er unternimmt verzweifelt weitere Intubationsversuche, die ebenfalls scheitern. Frau H. ist nun bradykard und drucklos. Beim Eintreffen des Anästhesisten ist sie asystolisch. Frau H. wird nun intubiert, stirbt jedoch an der Hypoxie.

Dr. S., ein Arzt mit hohem Verantwortungsbewusstsein, ist schwer traumatisiert und quält sich mit Selbstvorwürfen. Er spricht so oft mit seinen Kollegen über die Ereignisse, dass sie anfangen, ihm aus dem Weg gehen. Sie beginnen seine aus seinem Verantwortungsbewusstsein geborenen Selbstzweifel zu glauben und halten ihn für schuldig an dem Geschehen. Seine Vorgesetzten fürchten eine hohe Schadenersatzklage und wenden sich frühzeitig von ihm ab. Sie melden den Vorfall der Versicherung und weisen darauf hin, dass ein anderer Arzt die Patientin intubieren konnte, sodass hier offenbar das beklagenswerte Versagen eines Einzelnen vorläge. Dr. S. kündigt nach einigen Wochen, seine berufliche Entwicklung verläuft für Jahre nur noch schleppend.

Dieses Fallbeispiel ist nicht erfunden, leider.

\subsection{Fehler in der Notfallmedizin}

Untersucht man Fehler in verschiedenen Bereichen der Medizin, so zeigt sich schnell, dass manche fehlerträchtiger sind als andere. Auf Intensivstationen geschehen pro Patient mehr Fehler als auf Normalstationen (Wachter 2012). Notfallmedizin scheint ein besonders risikoträchtiger Bereich der Medizin zu sein. Aufgrund der besonderen und von anderen Gebieten sehr unterschiedlichen Bedingungen dieser Tätigkeit, z.B. viele und unbekannte Patienten, anfangs wenige zuverlässige Informationen, 
manchmal hohe Krankheits- oder Verletzungsschwere, nicht selten rasche Verschlechterungen, oft erhebliche Unklarheiten und atypische Präsentationen zu Beginn des Notfallgeschehens, erscheint dies gut nachvollziehbar (Cosby u. Croskerry 2009). Dabei ist zu berücksichtigen, dass ein Teil der Fehler in der Notfallmedizin, vielleicht sogar die Mehrheit, unerkannt bleiben dürfte. Vor allem in der präklinischen Notfallmedizin könnte ein ungünstiger Behandlungsverlauf nicht einem Fehler, sondern dem zugrund liegenden Krankheitsgeschehen zugeschrieben werden. Die Risiken und die Fehlerträchtigkeit der Tätigkeit in der Notfallmedizin sind aufgrund der dort herrschenden besonderen Bedingungen offenbar so hoch, dass einer der Pioniere der Patientensicherheits-Forschung die Notaufnahme als „natural laboratory for the study of error" bezeichnet hat (Croskerry u. Sinclair 2001).

Über die tatsächliche Häufigkeit von Fehlern im Rettungsdienst ist nur wenig bekannt (St. Pierre u. Hofinger 2014). Fehlermeldeportale, wie z.B. www.CIRS-Notfallmedizin.de, erfreuen sich eines hohen Zuspruchs, lassen aber aus methodischen Gründen keinen Rückschluss auf die Häufigkeit kritischer Ereignisse im Verhältnis zur Gesamtzahl aller Rettungsdiensteinsätze zu (Hohenstein et al. 2011). Critical Incident Reporting Systeme, CIRS, geben somit zwar keine Hinweise auf die tatsächliche Häufigkeit, wohl aber auf die Art der kritischen Ereignisse und geben Einsichten in die Fehler, die ihnen zu Grund liegen.

Bei der Analyse von fast 1.000 kritischen Ereignissen im Rettungsdienst zeigte sich, dass deren Ursache in $56 \%$ der Fälle auf das Verhalten der Helfer zurückzuführen war, während Materialprobleme nur in 20\% der Fehler auftraten (Chaudhary et al. 2014). Ein nicht unerheblicher Teil der Materialprobleme kann jedoch z.B. auf mangelhafte Wartung zurückgeführt werden, sodass mittelbar auch hier Menschen als Verursacher beteiligt sind.

Analysiert man die Fehler, die auf das Verhalten der Helfer im Rettungsdienst zurückzuführen sind, so zeigt sich, dass 66\% dieser kritischen Ereignisse durch unzureichende Kenntnisse und Erfahrung der Helfer entstehen (Chaudhary et al. 2014). Weitere Ursachen sind unzureichende Beherrschung der Arbeitstechniken (14\%) und Kommunikationsprobleme (13\%) (Chaudhary et al. 2014).

Fehler in der klinischen Notfallmedizin, den Notaufnahmen, sind besser untersucht als Fehler im Rettungsdienst (Cosby u. Croskerry 2009; Croskerry u. Sinclair 2001; Fordyce et al. 2003). In einer großen Beobachtungsstudie an fast 2.ooo Patienten einer US-amerikanischen Notaufnahme fanden sich Fehler bei 18\% der Patienten (Fordyce et al. 2003). Diese Rate liegt höher als bei Klinik-Patienten auf Normalstation (Wachter 2012). 22\% der beobachteten Fehler betrafen diagnostische Prozeduren, 16\% die Gabe von Medikamenten und 16\% administrative Prozesse (Fordyce et al. 2003).

98\% der Irrtümer im Emergency Department hatten jedoch keine schweren Folgen für den Patienten (Fordyce et al. 2003). Dies könnte im präklinischen Bereich anders sein. Nach einer vor kurzem erschienenen Studie hatten 15\% der im Rettungsdienst geschehenen Fehler eine negative Auswirkung auf den Zustand des Patienten oder den Behandlungserfolg, 78\% dieser Fehler hatten ihre Ursache bei den Helfern (Hohenstein et al. 2014).

Offenbar sind Fehler in der Notfallmedizin ein Thema, für die Patienten und für ihre Helfer. Fehler mögen in der Notfallmedizin vielleicht häufiger auftreten als in anderen Bereichen der Medizin, die ethische Beurteilung des Umgangs mit ihnen unterscheidet sich aber nicht, die ethischen Maßstäbe sind die gleichen. 
Fehler sind in der Notfallmedizin offenbar nicht selten und sie belasten Patienten und ihre Helfer.

\subsection{Ethischer Umgang mit Fehlern Patienten und Angehörigen gegenüber}

Der ethische Anspruch, die Wahrheit zu sagen, ist in unserer Kultur hoch angesiedelt. Er ist besonders hoch im Gesundheitswesen und gerade von Ärzten wird erwartet, dass sie die Wahrheit sagen. Dies zeigt sich auch in rechtlichen Normen. So zwingt das Patientenrechtegesetz im $\$ 630$ BCB Ärzte dazu, Patienten vom Verdacht auf das Vorliegen eines Behandlungsfehlers zu informieren, wenn dadurch eine Gesundheitsgefahr abgewendet werden kann. Dies gilt nicht nur für Fehler, die ein Arzt selbst begangen hat, sondern auch dann, wenn ein Arzt einen Fehler eines anderen Arztes bemerkt.

Dennoch ist es für Ärzte offenbar oft schwer, Fehler zu offenbaren. Dafür gibt es eine Reihe von Gründen (Moskop et al. 2006). So ist oft ist nicht vollkommen klar, ob wirklich ein Behandlungsfehler vorliegt. Ein weiterer wichtiger Grund könnte sein, dass bei vielen Helfern eine hohe Motivation zum Helfen vorliegt und es als schwer erträglich empfunden wird, doch einen Fehler begangen zu haben (Moskop et al. 2006). Ein an sich selbst oder von Kollegen gestellter Anspruch auf Fehlerlosigkeit begünstigt dann eher das Wegsehen als das Offenbaren. Auch die Hierarchie innerhalb der Medizin scheint ein Ansprechen von Fehlern eher zu behindern (St. Pierre et al. 2012). Weitere Widerstände können in der Angst vor der Reaktion der Patienten und ihrer Angehörigen, vor dem Verlust des Ansehens bei Kollegen, aber auch bei Freunden und Familie liegen (Moskop et al. 2006). Hinzu kommt Furcht vor rechtlichen Konsequenzen und inzwischen auch die Sorge vor dem Verlust des Versicherungsschutzes.

Alle diese Sorgen sind menschlich gut nachvollziehbar. Analysiert man die Hindernisse gegenüber dem Offenlegen eines Fehlers, so wird jedoch schnell klar, dass sie dem Interesse des Helfers dienen und nicht dem Patienten. Das Dilemma der Abwägung zwischen Eigenschutz und Ehrlichkeit ist zwar menschlich verständlich, aber ethisch meist auflösbar: Das höhere Gewicht dürfte in der Regel bei der Wahrheit liegen. Es ist nicht selten Kennzeichen eines ethischen Konfliktes, dass zwei Werte im konkreten Fall nicht vereinbar sind und ein moralisch gebotenes Verhalten, z.B. die Wahrheit sagen, zu negativen Konsequenzen, z.B. Haftung oder Strafe, für den Handelnden führen kann.

Für den betroffenen Helfer, der den Mut zum ethisch richtigen Vorgehen hat, wünscht man sich vor allem bei gravierenden Fehlerfolgen persönliche Eigenschaften und Werte, die den Betroffenen stabilisieren, und ein schützendes Umfeld durch Freunde und Familie. Wichtig sind aber auch Vorgesetzte, Kollegen und Mitarbeiter, die mehrere Tatsachen beachten, zum Beispiel:

- dass das Wohl des Patienten das höchste Gut ist

- dass ein Fehler auch ihnen selbst passieren kann

v dass künftigen Patienten am meisten dadurch geholfen werden kann, dass man den Fehler analysiert und Maßnahmen gegen eine Wiederholung einleitet

- dass auch ein Helfer durch einen Fehler sehr leiden kann. 


\section{Patienten und Angehörige haben ein ethisches und juristisches Recht auf die Offenlegung eines Fehlers.}

\subsection{Ethischer Umgang mit Fehlern Helfern gegenüber}

Fehler und Fehlerfolgen belasten in erster Linie die Patienten und ihre Angehörigen. Da aber zwischen Leid nicht wirklich abgewogen werden kann, sollte nicht außer Acht gelassen werden, dass auch Helfer unter Fehlern leiden, die sie begangen haben oder die ihnen zugeschrieben werden. Das Leiden der Helfer hängt dabei sowohl von der Schwere des Fehlers und seiner Folgen als auch von der Persönlichkeit des Helfers ab. Die Folgen eines Fehlers können für den Helfer sehr schwer sein, und reichen von Gefühlen der Schuld, Scham oder Hilfslosigkeit über Depression und Verzweiflung bis hin zum Suizid (Wulf 2013). Weitere Folgen, wie Beeinträchtigung der Entscheidungsfähigkeit und erhöhte Ängstlichkeit, erhöhen die Wahrscheinlichkeit für erneute Fehler. Andere Folgen wie Alkohol- und Medikamenten-Abhängigkeit, Wechsel des Krankenhauses, der Tätigkeit oder Berufsaufgabe sind ebenfalls bekannt (Wulf 2013).

Kollegen und vor allem Vorgesetzten des betroffenen Helfers kommt insbesondere bei schweren Fehlern und Fehlerfolgen große Bedeutung zu: Sie können seine Traumatisierung verstärken oder sie mindern. Diese Rolle kommt ihnen zu, ob sie dies wollen oder nicht. Dies ist ihnen aber vielfach nicht bewusst. Dazu kommt, dass sie oft auf diese Aufgabe auch nicht vorbereitet sind. Der Umgang mit Fehlern ist in der Regel weder Thema der Weiterbildung noch der Vorbereitung für Führungsaufgaben. Angesichts der Häufigkeit und Bedeutung von Fehlern in der Medizin ist dies schwer nachvollziehbar. Das Wissen, wie man nach einem Fehler ethisch, psychologisch und juristisch angemessen vorgehen könnte, um Patienten und betroffenen Helfern gleichermaßen gerecht zu werden, ist jedoch durchaus vorhanden: Es gibt sehr gute Empfehlungen sowohl für Kollegen als auch für Vorgesetzte im Gesundheitswesen zum ethischen, psychologischen und juristischen Vorgehen nach einem Fehler (Wulf 2013). Man muss sich allerdings schon vorher mit ihnen vertraut gemacht haben, um nach einem Fehler sofort auf sie zurückgreifen zu können.

Es sind vor allem zwei ethische Aspekte, die beim Umgang mit Helfern, die einen Fehler begangen haben, berücksichtigt werden müssen: Gerechtigkeit und Barmherzigkeit. Gerechtigkeit bedeutet in diesem Zusammenhang, dass ein Personalisieren von Schuld vermieden werden muss. Dem liegt die Erkenntnis zu Grund, dass bei der großen Mehrzahl der Fehler nicht ein Mensch versagt hat, sondern ein System (Reason 2000). Fehler können auch dadurch geschehen, dass Personen von Vorgesetzten mit einer Verantwortung betraut werden, der sie noch nicht gewachsen sind (s.a. Fallbeispiel 1). Dies ist im Gesundheitswesen nicht vollkommen ungewöhnlich.

Manchmal überhöhen stark von Verantwortungsgefühl geprägte Helfer ihren Anteil an der Schuld mehr als dies angemessen ist, oder ihnen wird die Schuld sogar zugeschoben. Dann müssen sie vor sich und vielleicht auch vor anderen geschützt werden.

In all diesen Varianten kann die Einhaltung des ethischen Prinzips der Gerechtigkeit gefordert sein. Wie weit die Pflicht von Beobachtern und Vorgesetzten geht, sich für Gerechtigkeit einzusetzen, bleibt offen für eine intensive Betrachtung. 
Sieht man, wie sehr gerade hochmotivierte und verantwortungsbewusste Helfer insbesondere bei schweren Fehlerfolgen leiden, und wie alleine gelassen sie sich oft nicht nur fühlen, sondern auch tatsächlich sind, liegt der christliche Wert der Barmherzigkeit sehr nahe. Mitgefühl mit Patienten und Helfern zu haben, Verständnis und Empathie für beide zu zeigen, sind hochstehende ethische Ansprüche in Situationen wie diesen. Manchmal lässt sich dies nach Fehlern in der Medizin in beeindruckender Weise beobachten, nicht ganz selten aber auch nicht.

Nicht nur Patienten, auch Helfer verdienen nach einem Fehler Gerechtigkeit und Barmherzigkeit.

\subsection{Ethischer Umgang mit Fehlern künftigen Patienten gegenüber}

Bei Experten für Patientensicherheit ist unumstritten, dass die große Mehrzahl der Fehler in der Medizin nicht auf das Versagen einzelner Personen zurückzuführen ist, sondern auf das Versagen einzelner Bestandteile eines Systems (Croskerry u. Sinclair 2001; Rall et al. 2001; Reason 2000). Dies ist in der Notfallmedizin nicht anders als in anderen Bereichen der Medizin (Croskerry u. Sinclair 2001).

Wird diese Erkenntnis missachtet und die Schuld am Fehler einer einzelnen Person zugeschoben, sie also personalisiert, dann vergibt man die Chance, den Fehler auf der Systemebene $z u$ analysieren und dort Maßnahmen $z u$ treffen, die wesentlich wirkungsvoller sind als die Belehrung oder das Austauschen einer einzelnen Person (Croskerry u. Sinclair 2001; Rall et al. 2001; Reason 2000).

Die wahre Ursache vieler Fehler, die in der Medizin geschehen, liegt oft wesentlich tiefer als es auf den ersten Blick den Anschein hat. Es gibt standardisierte Verfahren, wie die Root Cause Analysis, die diese tiefen Ursachen aufdecken können. Wird auf dieser Ebene, der Systemebene, reagiert, dann sinkt die Wahrscheinlichkeit oft ganz erheblich, dass sich der Fehler wiederholt. Wird dies jedoch unterlassen, dann kann es geschehen, dass spätere Patienten unter dem gleichen Fehler zu leiden haben, der sich erneut verwirklicht hat, nur mit anderen Personen.

Somit besteht vor allem für die Vorgesetzten im Gesundheitswesen die ethische Verpflichtung, Fehler zur Kenntnis zu nehmen und zu analysieren, und Maßnahmen zu ergreifen, die die Wahrscheinlichkeit ihrer Wiederholung vermindern. Dazu ist jedoch erhebliche Sachkenntnis im Risk Management erforderlich.

Damit gelingt es aber, eine ,Safety Culture‘ einzuführen, die das Gesundheitswesen auch in diesem Bereich zu einer rasch lernenden Organisation machen kann (Croskerry u. Sinclair 2001; Rall et al. 2001; Reason 2000). Künftigen Patienten bleiben dann manche Fehler erspart, die bereits anderswo geschehen sind. In einem weiteren Sinne dient dieses Vorgehen hochstehenden ethischen Prinzipien, wie dem ,primum non nocere“ von Scribenius Largus, der Nächstenliebe kommenden Patienten gegenüber und dem Schutz von Menschen, die ihren Helfern in einer Notfallsituation vertrauen. 


\section{Fallbeispiel 1 - Fortsetzung}

Durch seine Kündigung erfährt Dr. S. nicht mehr, dass nach einigen Monaten Dr. H., ein erfahrener Risk Manager, als Berater in die Klinik kommt, da der Versicherung inzwischen mehrere Behandlungsfehler gemeldet werden mussten. Dr. H. analysiert auch diesen Fall und fragt nach, ob es in der Klinik eine Überprüfung gäbe, ob ein junger Arzt bereits erfahren genug sei, um allein in der Notaufnahme Dienst zu tun. Dies ist nicht der Fall. Er fragt weiter, ob es ein Training für kritische Ereignisse wie dieses gäbe. Auch dies ist nicht der Fall. Es gibt auch keine Hinzuziehungsregeln, wann ein erfahrener Arzt gerufen werden muss. Ebenso fehlt im Haus ein klares Konzept, wie bei einem bedrohten Atemweg vorzugehen sei. Dr. H. weist darauf hin, dass all diese Bedingungen zur Gewährleistung der Patientensicherheit von den Leitenden Ärzten zu erfüllen sind, und nicht vom diensthabenden Arzt.

In den folgenden Wochen werden alle diese Voraussetzungen geschaffen. Die Wiederholung eines solchen Ereignisses wird dadurch wesentlich weniger wahrscheinlich.

\section{Die Analyse von Fehlern und Maßnahmen zu ihrer Vermeidung dienen dem Schutz künftiger Patienten.}

\subsection{Ausblick}

Betrachtet man den Umgang mit Fehlern im Gesundheitswesen, so wird schnell klar, dass ethische Werte wie Ehrlichkeit, Gerechtigkeit, Barmherzigkeit und Fürsorge große Bedeutung haben. Ebenso schnell wird klar, dass diese Werte nicht nur für die betroffenen Patienten und Angehörigen wichtig sind, sondern auch für die Helfer, seien sie direkt oder mittelbar betroffen. Die Werte und ethischen Prinzipien unterscheiden nicht nach den Personengruppen.

Man muss zur Kenntnis nehmen, dass die Tätigkeit in der Notfallmedizin, im Rettungsdienst und in Notaufnahmen aufgrund ihrer besonderen Charakteristika offenbar besonders fehlerträchtig und risikobehaftet ist. Vielleicht ziehen die besonderen Herausforderungen dieser Tätigkeit gerade deshalb auch besonders verantwortungsbewusste Menschen mit einem hohen Anspruch an die eigene Leistung an. Dies begünstigt dann aber auch eine Traumatisierung, wenn ihnen dennoch Fehler unterlaufen.

Patienten, Helfern, Vorgesetzten in der Notfallmedizin, eigentlich dem gesamten Gesundheitswesen, wünscht man Sensibilität im Umgang mit Fehlern, eine Abkehr vom Personalisieren von Schuld und eine Hinwendung zur Schaffung fehlerarmer Systeme, und vor allem die Einhaltung ethischer Prinzipien auf all diesen Ebenen.

\section{Literatur}

Chaudhary T, Fleischmann T, Schultheis K, Hohenstein C (2014) Möglichkeiten eines CIRS in der präklinischen Notfallmedizin. Notarzt 30: 2-6

Cosby K, Croskerry P (2009) The Nature of Emergency Medicine. In: Croskerry P, Cosby K, Schenkel S, Wears R (Hrsg.)

Patient Safety in Emergency Medicine. Wolters Kluwer Lippincott Williams and Wilkins, S. 5-7

Croskerry P, Sinclair D (2001) Emergency medicine: A practice prone to error? CJEM 3: 271-276 
Fordyce I, Blank FS, Pekow P, Smithline HA, Ritter G, Gehlbach S, Benjamin E, Henneman PL (2003) Errors in a busy emergency department. Annals of Emergency Medicine 42: 324-333

Hohenstein C, Fleischmann T, Hempel D (2011) Kritische Ereignisse in der Notfallmedizin. Notfallmedizin up2date 6: 89-104

Hohenstein C, Hempel D, Schultheis K, Lotter O, Fleischmann T (2014) Critical incident reporting in emergency medicine: results of the prehospital reports. Emerg Med I 31: 415-418

Moskop IC, Geiderman IM, Hobgood CD, Larkin GL (2006) Emergency physicians and disclosure of medical errors. Ann Emerg Med 48: 523-531

Rall M, Manser T, Guggenberger H, Gaba DM, Unertl K (2001) Patientensicherheit und Fehler in der Medizin. AINS 36: $321-330$

Reason I (2000) Human error: models and management. BMJ 320: 768-70

St. Pierre M, Hofinger G (2014) Human Factors und Patientensicherheit in der Akutmedizin. Springer-Verlag, Berlin

St. Pierre M, Scholler A, Strembski D, Breuer G (2012) Äußern Assistenzärzte und Pflegekräfte sicherheitsrelevante Bedenken? Anaesthesist 61: 857-66

Wachter R (2012) Understanding Patient Safety. McGrawHill, New York

Wulf H (2013) Patientensicherheit - Auftrag für die Zukunft. Empfehlungen zum Umgang mit schweren Behandlungskomplikationen und belastenden Verläufen. AINS 54: 490-494

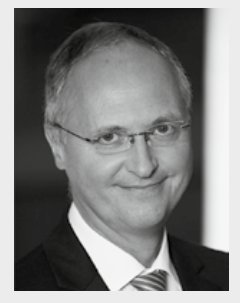

\section{Dr. med. Thomas Fleischmann, EBCEM, FESEM, FRCEM, MHBA}

Studium der Humanmedizin in Erlangen. Weiterbildung in Chirurgie und Innerer Medizin in Nürnberg. Facharzt für Allgemeinmedizin, Zusatzbezeichnungen Notfallmedizin, Klinische Notfallmedizin SGNOR (CH), Ärztliches Qualitätsmanagement. Seit 25 lahren nur in Notaufnahmen und Notarztdienst tätig. Einer der ersten Europäischen Fachärzte für Notfallmedizin (European Board Examination in Emergency Medicine), Fellow der European Society for Emergency Medicine EuSEM und Fellow by Election des Royal College of Emergency Medicine RCEM (UK). 